\title{
Misdiagnosed schwannoma of the medial plantar nerve of the foot
}

\begin{abstract}
Metatarsalgia is a condition of the foot characterized with pain, numbness or burning feeling located in the forefoot or midfoot region. Metatarsalgia is often referred to as a symptom, rather than as a specific disease. Common causes of metatarsalgia include neuroma between the toes (also known as Morton's neuroma), metatarsophalangeal synovitis, avascular necrosis, sesamoid bone inflammation and inflammatory arthritis. Other conditions such as tarsal tunnel syndrome can also present with pain or numbness in the metatarsal region.

We present a case of a patient with Schwannoma in atypical localization- at the medial plantar nerve of the foot causing severe metatarsalgia. The patient was treated conservatively without any success. She underwent tarsal tunnel release surgery without sufficient radiological examinations for differentiation of the clinical condition. After making the necessary MRI exam in our institution, we confirmed the Schwannoma at the medial plantar nerve of her left foot. A neurectomy of the nerve proximal to the tumor was done, which resolved the clinical symptoms.
\end{abstract}

Keywords: Metatarsalgia, Schwannoma, Medial plantar nerve
Volume 9 Issue I - 2017

\author{
Milan Samardziski,' Neron Popovski,' Nenad \\ Atanasov, ${ }^{2}$ Nikola Gramatnikovski,' Ilir \\ Shabani' \\ 'University Clinic for Orthopedic Surgery, Macedonia \\ ${ }^{2}$ University Clinic for thoracic and vascular surgery, Macedonia
}

Correspondence: Milan Samardziski, University Clinic for thoracic and vascular surgery, Skopje, Macedonia Email Milan_samardziski@yhoo.com

Received: April 05, 2017| Published: September 20, 2017

\section{Introduction}

Metatarsalgia is a condition defined as a compression syndrome mechanically induced by intrinsic and extrinsic biomechanical factors that result in sharp pain, paresthesia and in some cases numbness of the forefoot. Metatarsalgia is often referred to as a symptom, rather than as a specific disease. Common causes of metatarsalgia include neuroma between the toes (also known as Morton's neuroma), metatarsophalangeal synovitis, avascular necrosis, sesamoiditis, and inflammatory arthritis. ${ }^{1}$ Sometimes pain irradiates in the midfoot or in the whole foot, making difficult to distinguish other problems such as: tarsal tunnel syndrome, neuroma or schwannoma of the foot nerves. We present a case of a patient with Schwannoma in atypical localization - the medial plantar nerve of the foot causing severe metatarsalgia.

A schwannoma is a tumor of the peripheral nervous system that arises in the nerve sheath, more specifically from a type of cell called a Schwann cell, giving the tumor its name. It is almost always benign. The most common locations include the head, arms, legs and trunk. The presence of a schwannoma may first become apparent with a cosmetic deformity, a noticeable mass, symptoms similar to a compressive neuropathy or some combination of these. Neurologic symptoms tend to occur later. ${ }^{1}$

Symptoms can be vague, and it may be several years before the tumor is diagnosed. ${ }^{1}$ The cause of schwannomas is not known. In some cases they are associated with neurofibromatosis. ${ }^{1,2}$ In these cases, multiple tumors may be present. ${ }^{2}$ Treatment of benign schwannomas involves surgery to remove the tumor, which usually results in complete and fast relief of symptoms. ${ }^{1}$ Schwannomas very rarely become malignant in which cases the treatment may involve surgery, radiation therapy, and/or chemotherapy.

\section{Case report}

A 50-year-old woman, presented to the University Clinic for Orthopedic Surgery in Skopje, R. Macedonia with severe, sharp, disabling pain on the medial plantar side of her left foot irradiating to the first, second and third toes, prone to aggravation with certain activities, and diminishing with rest. She had no pain while in bed at night. There were no obvious anatomical anomalies. She experienced these symptoms two years prior to this hospitalization. Following the advice of her GP she started using analgesic medications that did not give any result. She consulted an orthopedic surgeon in a different institution. She was diagnosed as having a tarsal tunnel syndrome based on the history of her condition, clinical examination and (only) $\mathrm{x}$-ray. Physical therapy and analgesic medications were suggested. The patient underwent two cycles of physical therapy in the following year without any changes of the condition. Since the conservative treatment did not give any result, a surgical treatment was suggested. She had a tarsal tunnel release surgery.

The operative and postoperative period passed without complications but she continued to have the same problems after the surgery. The patient received analgesic medications few months before she came to our Clinic. On her first visit at our outpatient clinic in July 2008, orthopedic shoes made on a plaster model of her feet with metatarsal pad were prescribed. On the control examination, the patient complained that the symptoms were worse and even disabling. An urgent MRI of the left foot was advised. The MRI showed a 2.5-centimeter in diameter, round, encapsulated mass that was differentially diagnosed as lipoma or neuroma (Figure 1a-1d). A surgical extirpation of the tumor was suggested. The operation was performed in September 2009 at the University Clinic for Orthopedic Surgery. At the level of the medial cuneiform bone of the foot, a round encapsulated mass, surrounding the medial communicant branch of medial plantar nerve was found (Figure 1e). Since the tumor could not be detached from the nerve, a neurectomy of the nerve proximal to the tumor was done. There were no complications in the operative and postoperative period. The excised tissue was sent for histological examination and the result confirmed it to be a Schwannoma (Figure 1f). After surgery, the patient was relieved of all symptoms. Followup visits were carried out at three, six and twelve months after the excision of the Schwannoma. The patient had no pain and function of the left foot was normal at each consecutive control. 

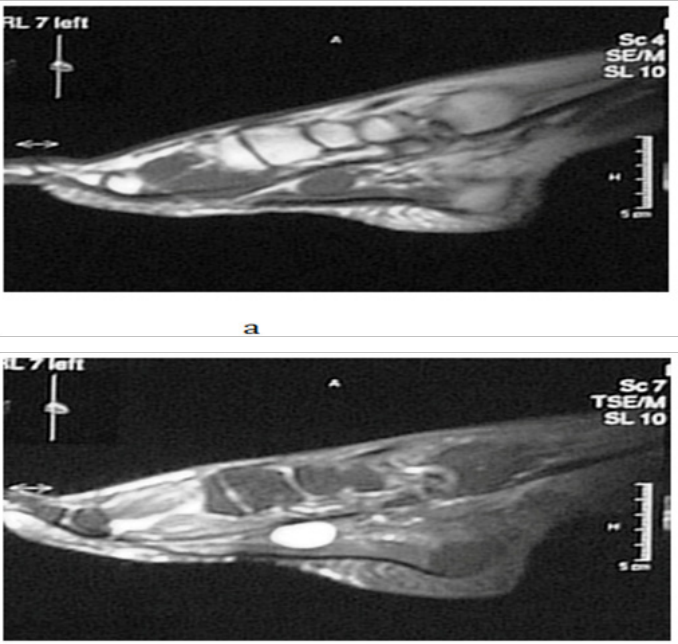

b

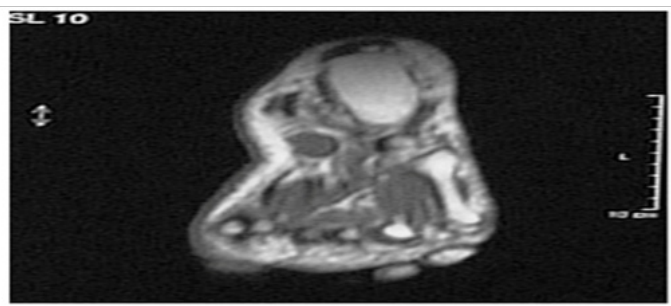

$c$

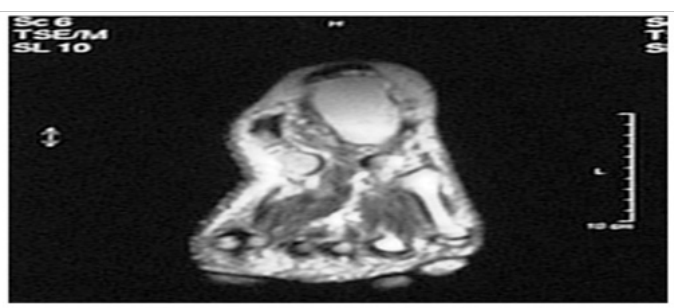

d

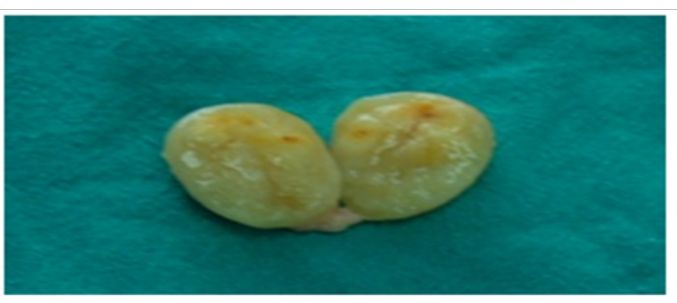

e

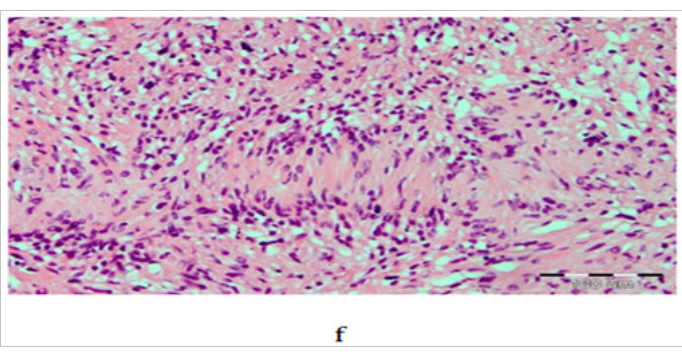

Figure I a, b) MRI cross section of the foot in TI and T2 sequence (arrow shoving Schwannoma), c, d) MRI transverse section of the foot in TI and T2 sequence e) macroscopic appearance of excised and cut in half Schwannoma f) Verocay body surrounded by Anthony $B$ cells $(\mathrm{HE}, x \mathrm{IO0})$.

\section{Discussion}

The tibial nerve enters the tarsal tunnel and divides it into a medial and a plantar nerve. They accompany their vascular supply separated only by a thin membrane. Having passed the medial (upper) canal of arterial and venous lacuna of the tarsal tunnel, the nerve lies behind the vessels situated above the abductor muscle of the first toe. The nerve crosses below the talus and the navicular bone, and then crosses the lateral margin of the abductor hallucis muscle lying between the fibro-muscular part of the muscle and the navicular tuber. In this region the medial plantar nerve is lying within the bone and fibromuscular tunnel, where it can often be compressed. ${ }^{3-5}$ The symptoms in our patient were consistent with medial plantar nerve syndrome described as Jogger's foot in 1978 by Rask. ${ }^{6}$

The patient had a positive Tinel's sign and the symptoms aggravated with the orthopedic shoes that were made by a model of her feet. ${ }^{3,6}$ These two facts in most cases lead to Morton's neuroma as differential diagnosis, but in our case the severity of the symptoms that were near to disabling and the non-responsiveness to physical therapy led to a condition more serious than irritation of the medial plantar nerve. ${ }^{7,8}$ Thus, an MRI of the patient's left foot was advised, which proved to be the key for correct treatment and diagnosis of this condition. There can be more than one cause of metatarsalgia, including: interdigital neuroma (also known as Morton's neuroma), metatarsophalangeal synovitis, avascular necrosis, sesamoiditis, and inflammatory arthritis. ${ }^{8}$ Other conditions such as tarsal tunnel syndrome can also present with pain or numbness in the metatarsal region. Metatarsalgia is a condition that occurs relatively frequently. Schwannoma of the medial plantar nerve is relatively rare condition, resulting in pain and numbness of the innervating area of the nerve. Often pain irradiates in mid-front or/and in posterior part of the foot, mimicking some of the former mentioned conditions. Due to this, in diagnosis and treatment of Schwannoma of the foot, detailed physical examination and appropriate MRI scans are essential.

\section{Acknowledgments}

None.

\section{Conflicts of interest}

None.

\section{References}

1. Dickey ID Neurilemmoma. Medscape. 2016

2. Neurofibromatosis type 2. Genetic Home. 2013

3. Marko Pecina, Jelena Krmpotic-Nemanic, Andrew Markiewitz Tunnel Syndromes: peripheral nerve compression syndromes. ( $\left.3^{\text {rd }} e d n\right)$, Boca Raton, Florida, USA, pp. 2000;267-271.

4. Hughes RJ, Ali K, Jones $\mathrm{H}$ et al. Treatment of Morton's neuroma with alcohol injection under sonographic guidance: follow-up of 101 cases. AJR Am J Roentgenol. 2007;188(6):1535-1539.

5. Wu KK Morton's interdigital neuroma: a clinical review of its etiology, treatment, and results. J Foot Ankle Surg. 1996;35(2):112-119.

6. Rask MR Medial plantar neuropraxia (jogger's foot). Report of 3 cases. Clin Orthop. 1978;134:193-195.

7. Kim JY, Choi JH, Park J et al. An anatomical study of Morton's interdigital neuroma: the relationship between the occurring site and the deep transverse metatarsal ligament (DTML). Foot Ankle Int. 2007;28(9):1007-1010.

8. Morscher E, Ulrich J, Dick W Morton's intermetatarsal neuroma: morphology and histological substrate. Foot Ankle Int. 2000;21(7):558-562. 